\title{
Nonlinear interactions between relativistic radiation belt electrons and oblique whistler mode waves
}

\author{
X. Tao and J. Bortnik \\ Department of Atmospheric and Oceanic Sciences, University of California, Los Angeles, California, USA
}

Received: 30 June 2010 - Revised: 28 August 2010 - Accepted: 9 October 2010 - Published: 26 October 2010

\begin{abstract}
Resonant interactions between relativistic charged particles and oblique whistler mode waves are explored in this work, and it is shown that nonlinear phase trapping could happen in a gyrophase averaged sense, consistent with previous studies of interactions between nonrelativistic electrons and oblique whistler mode waves. A dimensionless parameter $\chi$ is derived to represent the ratio of wave-induced motion to the adiabatic motion of the particle. We show that phase trapping is likely to occur when the wave-induced motion dominates the adiabatic motion, which is caused mainly by the background fields. A mapping of probable regions of nonlinear interactions is shown based on the parameter $\chi$. We show that the nonlinear interactions might be important near the equatorial plane for even moderate wave amplitude, and the latitudinal range for nonlinear interactions to occur is largest for electrons with local pitch angles around 50 degrees, consistent with previous findings. The results are important for understanding the nonlinear dynamics of relativistic radiation belt electrons and the generation of chorus waves.
\end{abstract}

\section{Introduction}

The Earth's outer radiation belt contains relativistic electrons and is very dynamic during disturbed times; electron fluxes could vary by several orders of magnitude (Reeves et al., 2003; Friedel et al., 2002), making the radiation belt hazardous to spacecraft and astronauts (Baker et al., 1994, 1997). Interactions between electrons and whistler mode waves have been established as one of the main mechanisms for controlling the dynamics of Earth's radiation belts from both observation and simulation (Horne and Thorne, 1998;

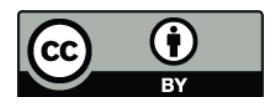

Correspondence to: $\mathrm{X}$. Tao

(xtao@atmos.ucla.edu)
Horne et al., 2005a,b; Bortnik and Thorne, 2007; Furuya et al., 2008; Varotsou et al., 2008; Albert et al., 2009). While most of the previous research on wave particle interactions in the outer radiation belt is based on the quasilinear theory, nonlinear interactions between electrons and whistler mode waves are considered to be important in two aspects.

First, recent observations of large amplitude whistler mode chorus waves (Cattell et al., 2008; Cully et al., 2008) bring question to the validity of using quasilinear theory to describe effects of chorus waves on electron dynamics. Quasilinear theory was developed to describe interactions between charged particles and small amplitude broad band waves (Kennel and Engelmann, 1966). Even though a small amplitude monochromatic wave could be shown to give the same diffusion coefficients as a narrow-band limit of quasilinear theory when nonlinear effects such as phase trapping and bunching are not important (Albert, 2010), increased amplitude of waves can introduce strong nonlinear effects and bring the use of quasilinear theory into question (Bortnik et al., 2008).

Second, chorus waves are ELF/VLF whistler mode waves consisting of rising or falling tones seen at a fixed location (Santolík et al., 2003; Inan et al., 2004; Breneman et al., 2009; Chum et al., 2009). The generation mechanism of chorus waves has been an outstanding question for some time (Omura et al., 1991). Several mechanisms involving nonlinear interactions between electrons and waves have been proposed to explain the formation of rising and falling tones (Helliwell, 1967; Nunn et al., 1997; Omura et al., 2008, 2009). Omura et al. (2008) derived a simple equation for calculating chorus wave frequency sweep rates based on the inhomogeneity ratio for nonlinear interactions between relativistic electrons and a parallel propagating wave. Other proposed mechanisms for chorus wave generation include the backward wave oscillator model (Trakhtengerts, 1995) among others (Omura et al., 1991). 
The criterion for nonlinear interactions between parallel propagating whistler mode waves and nonrelativistic electrons has been derived by Dysthe (1971) and relativistic electrons by Omura et al. (2008) from the Lorentz equations of motion. The inhomogeneity parameter for nonrelativistic electrons and oblique whistler mode waves has been derived by Bell (1984). Bell (1984) shows that while phase trapping could not exist in the ordinary sense as is the case of parallel propagating waves, it does exist in a gyro-averaged sense. A similar criterion has also been derived using Hamiltonian dynamics (Albert, 1993). In this article, we generalize the theory of Bell (1984) to the relativistic case to explore nonlinear interactions between relativistic radiation belt electrons and oblique whistler mode waves.

We start from the relativistic Lorentz equations of motion and perform a linear analysis to write the equations in $\left(p_{\perp}, p_{\|}, \zeta\right)$ coordinates in Sect. 2. Here $p_{\perp}$ and $p_{\|}$are the momentum components perpendicular and parallel to the ambient magnetic field, respectively, and $\zeta$ is the phase angle between $\boldsymbol{p}_{\perp}$ and the right hand component of the perpendicular wave magnetic field. A dimensionless parameter $\chi$ is derived as a criterion for nonlinear interactions in Sect. 3. We then produce a map of $\chi$ as a function of latitude and wave normal angle for a simplified case in Sect. 4, and summarize our results in Sect. 5.

\section{Equations of motion in $\left(p_{\perp}, p_{\|}, \zeta\right)$ coordinates}

We choose a Cartesian coordinate system with $z$ axis along the background field direction. The background field is $\boldsymbol{B}_{0}=$ $B_{0 z} \boldsymbol{e}_{\mathrm{z}}+\boldsymbol{B}_{0 \perp}$, with $B_{0 \mathrm{z}}$ the dipole field strength at $z$, which is the length along its field line from the equatorial plane. We choose

$\boldsymbol{B}_{0 \perp}=-x \frac{\partial B_{0 \mathrm{z}}}{\partial z} \boldsymbol{e}_{\mathrm{x}}$,

so that $\nabla \cdot \boldsymbol{B}_{0}=0$. Note that $B_{0 \perp} / B_{0 \mathrm{z}} \sim \epsilon_{0} \equiv \rho_{\perp} / R$, with $\rho_{\perp}$ the particle's gyroradius and $R$ the planet radius. The above choice of the background field is equivalent to ignoring the curvature of the Earth's dipole field.

\subsection{Equations of whistler wave fields}

We now consider the interaction between a charged particle and a whistler mode wave. The whistler mode wave magnetic and electric fields with wave normal vector $\boldsymbol{k}=$ $k(\sin \psi, 0, \cos \psi)$ are given by

$$
\begin{aligned}
& \boldsymbol{B}_{\mathrm{w}}=\boldsymbol{e}_{\mathrm{x}} B_{\mathrm{x}}^{\mathrm{w}} \cos \Phi-\boldsymbol{e}_{\mathrm{y}} B_{\mathrm{y}}^{\mathrm{w}} \sin \Phi+\boldsymbol{e}_{\mathrm{z}} B_{\mathrm{z}}^{\mathrm{w}} \cos \Phi, \\
& \boldsymbol{E}_{\mathrm{w}}=-\boldsymbol{e}_{\mathrm{x}} E_{\mathrm{x}}^{\mathrm{w}} \sin \Phi-\boldsymbol{e}_{\mathrm{y}} E_{\mathrm{y}}^{\mathrm{w}} \cos \Phi-\boldsymbol{e}_{\mathrm{z}} E_{\mathrm{z}}^{\mathrm{w}} \sin \Phi,
\end{aligned}
$$

where $\Phi \equiv \int \boldsymbol{k} \cdot d \boldsymbol{r}-\int \omega d t$ is the wave phase angle, $\psi$ is the wave normal angle, and $\boldsymbol{e}_{i}$ is the unit vector of the $i$-th axis. Here the wave fields satisfy

$$
B_{\mathrm{x}} / B_{\mathrm{y}}=-\frac{D\left(P-n^{2} \sin ^{2} \psi\right)}{P\left(S-n^{2}\right)},
$$

$$
\begin{aligned}
& B_{\mathrm{z}} / B_{\mathrm{y}}=\frac{D \sin \psi\left(P-n^{2} \sin ^{2} \psi\right)}{P \cos \psi\left(S-n^{2}\right)}, \\
& E_{\mathrm{x}} / B_{\mathrm{y}}=\frac{c\left(P-n^{2} \sin ^{2} \psi\right)}{n P \cos \psi}, \\
& E_{\mathrm{y}} / B_{\mathrm{y}}=\frac{D c\left(P-n^{2} \sin ^{2} \psi\right)}{n P \cos \psi\left(n^{2}-S\right)}, \\
& E_{\mathrm{z}} / B_{\mathrm{y}}=-\frac{c n \sin \psi}{P},
\end{aligned}
$$

which can be derived from Maxwell equations $\boldsymbol{k} \times \boldsymbol{E}_{\mathrm{w}}=$ $\omega \boldsymbol{B}_{\mathrm{w}}$ and $\boldsymbol{n} \times\left(\boldsymbol{n} \times \boldsymbol{E}_{\mathrm{w}}\right)+\mathrm{K} \cdot \boldsymbol{E}_{\mathrm{w}}=0$ with $\boldsymbol{n} \equiv c \boldsymbol{k} / \omega$ the refractive index, and $\mathrm{K}$ the dielectric tensor (Stix, 1962, p. 10). Here $P, S$, and $D$ are the usual Stix parameters, defined in Stix (1962, p. 10), and $c$ is the speed of light in vacuum.

It is convenient to define the R-and L-components of the perpendicular wave magnetic field according to

$$
\begin{gathered}
\boldsymbol{B}_{\mathrm{R}}=\frac{B_{\mathrm{x}}^{\mathrm{W}}+B_{\mathrm{y}}^{\mathrm{W}}}{2}\left(\boldsymbol{e}_{\mathrm{x}} \cos \Phi-\boldsymbol{e}_{\mathrm{y}} \sin \Phi\right), \\
\boldsymbol{B}_{\mathrm{L}}=\frac{B_{\mathrm{x}}^{\mathrm{W}}-B_{\mathrm{y}}^{\mathrm{w}}}{2}\left(\boldsymbol{e}_{\mathrm{x}} \cos \Phi+\boldsymbol{e}_{\mathrm{y}} \sin \Phi\right),
\end{gathered}
$$

so that $\boldsymbol{B}_{\perp}^{\mathrm{w}}=\boldsymbol{B}_{\mathrm{R}}+\boldsymbol{B}_{\mathrm{L}}$. The relationship between $\boldsymbol{B}_{\mathrm{R}}, \boldsymbol{B}_{\mathrm{L}}$, and $\boldsymbol{p}_{\perp}$ is shown in Fig. 1. Similarly we define the R- and L-components of the perpendicular electric field as

$$
\begin{gathered}
\boldsymbol{E}_{\mathrm{R}}=\frac{E_{\mathrm{x}}^{\mathrm{w}}+E_{\mathrm{y}}^{\mathrm{w}}}{2}\left(-\boldsymbol{e}_{\mathrm{x}} \sin \Phi-\boldsymbol{e}_{\mathrm{y}} \cos \Phi\right), \\
\boldsymbol{E}_{\mathrm{L}}=\frac{E_{\mathrm{x}}^{\mathrm{w}}-E_{\mathrm{y}}^{\mathrm{w}}}{2}\left(-\boldsymbol{e}_{\mathrm{x}} \sin \Phi+\boldsymbol{e}_{\mathrm{y}} \cos \Phi\right),
\end{gathered}
$$

and $\boldsymbol{E}_{\perp}^{\mathrm{W}}=\boldsymbol{E}_{\mathrm{R}}+\boldsymbol{E}_{\mathrm{L}}$.

\subsection{The Lorentz equation of motion}

The relativistic Lorentz equation of motion is

$\dot{\boldsymbol{p}}=q\left[\boldsymbol{E}_{\mathrm{w}}+\frac{\boldsymbol{p}}{\gamma m} \times\left(\boldsymbol{B}_{\mathrm{w}}+\boldsymbol{B}_{0}\right)\right]$,

Here $q$ is the charge of the particle, $m$ its mass, and $\gamma \equiv$ $\sqrt{1+p^{2} / m^{2} c^{2}}$ the relativistic factor. Note that the sign of the charge is contained in $q$, so for an electron $q=-1.6 \times$ $10^{-19} \mathrm{C}$ in SI units.

We now write the Lorentz equation using coordinates $\left(p_{\perp}, p_{\|}, \zeta\right)$. Using $\theta \equiv \tan ^{-1}\left(p_{\mathrm{y}} / p_{\mathrm{x}}\right)$, the angle between $\boldsymbol{p}_{\perp}$ and $\boldsymbol{e}_{\mathrm{x}}$ (as shown in Fig. 1), we have the angle between $\boldsymbol{p}_{\perp}$ and $\boldsymbol{B}_{\perp}^{\mathrm{w}}$ as $\zeta=\theta+\Phi$. To the lowest order in $\epsilon \equiv\left|B_{\mathrm{w}}\right| / B_{0}$, which is about $10^{-5} \sim 10^{-3}$ for typical chorus waves observed in space (Bell, 1984; Tsurutani et al., 2009), we have

$\dot{\theta}=\frac{1}{1+\left(p_{\mathrm{y}} / p_{\mathrm{x}}\right)^{2}} \frac{\mathrm{d}}{\mathrm{d} t}\left(\frac{p_{\mathrm{y}}}{p_{\mathrm{x}}}\right)=-\Omega$,

where $\Omega \equiv q B_{0 z} / \gamma m$. Note that we have dropped the $\mathcal{O}(\epsilon)$ term in the above equation, because its gyro-averaged value 
is $\mathcal{O}\left(\epsilon^{2}\right)$ (Bell, 1984), thus the term will not contribute to the analysis in Sect. 3, where only terms up to $\mathcal{O}(\epsilon)$ are kept. Thus

$\dot{\zeta}=\dot{\theta}+\dot{\Phi}=-\Omega+k_{\|} v_{\|}+k_{\mathrm{x}} v_{\mathrm{x}}-\omega$

The equation describing $\dot{p}_{\|}$is

$\dot{p}_{\|}=q\left[\boldsymbol{E}_{\mathrm{w}}+\frac{\boldsymbol{p}}{\gamma m} \times\left(\boldsymbol{B}_{\mathrm{w}}+\boldsymbol{B}_{0}\right)\right] \cdot \boldsymbol{e}_{\mathrm{z}}$.

By using the vector relations between $\boldsymbol{p}_{\perp}$ and $\boldsymbol{B}_{\mathrm{R}}$ and $\boldsymbol{B}_{\mathrm{L}}$ as shown in Fig. 1 and keeping only first order terms in $\epsilon$ and $\epsilon_{0}$, it can be shown that

$\dot{p}_{\|}=-q E_{\mathrm{z}}^{\mathrm{w}} \sin \Phi-\omega_{\mathrm{R}} p_{\perp} \sin \zeta-\omega_{\mathrm{L}} p_{\perp} \sin (\zeta-2 \Phi)+A_{1},($

where $\omega_{\mathrm{R}, \mathrm{L}}=q B_{\mathrm{R}, \mathrm{L}}^{\mathrm{W}} / \gamma m$ and

$A_{1}=\left(\frac{q \boldsymbol{p}_{\perp}}{\gamma m} \times \boldsymbol{B}_{0 \perp}\right) \cdot \boldsymbol{e}_{\mathrm{z}}$.

The equation of $\dot{p}_{\perp}=q\left[\boldsymbol{E}_{\mathrm{w}}+(\boldsymbol{p} / \gamma m) \times\left(\boldsymbol{B}_{\mathrm{w}}+\boldsymbol{B}_{0}\right)\right] \cdot \boldsymbol{e}_{\perp}$, where $\boldsymbol{e}_{\perp} \equiv \boldsymbol{p}_{\perp} / p_{\perp}$, could be shown similarly to be

$\dot{p}_{\perp}=\left(p_{\|}-\kappa_{\mathrm{R}}\right) \omega_{\mathrm{R}} \sin \zeta+\left(p_{\|}+\kappa_{\mathrm{L}}\right) \omega_{\mathrm{L}} \sin (\zeta-2 \Phi)+A_{2}$,

where

$\kappa_{\mathrm{R}}=\gamma m \frac{E_{\mathrm{R}}^{\mathrm{w}}}{B_{\mathrm{R}}^{\mathrm{w}}}=\gamma m \frac{E_{\mathrm{x}}^{\mathrm{w}}+E_{\mathrm{y}}^{\mathrm{w}}}{B_{\mathrm{x}}^{\mathrm{w}}+B_{\mathrm{y}}^{\mathrm{w}}}$,

$\kappa_{\mathrm{R}}=\gamma m \frac{E_{\mathrm{L}}^{\mathrm{w}}}{B_{\mathrm{L}}^{\mathrm{w}}}=\gamma m \frac{E_{\mathrm{x}}^{\mathrm{w}}-E_{\mathrm{y}}^{\mathrm{w}}}{B_{\mathrm{x}}^{\mathrm{w}}-B_{\mathrm{y}}^{\mathrm{w}}}$,

and

$A_{2} \equiv \frac{q}{\gamma m}\left(\boldsymbol{p} \times B_{0}\right) \cdot \boldsymbol{e}_{\perp}$.

\section{Conditions for nonlinear interactions}

As shown by Bell (1984) for the nonrelativistic case, phase trapping could happen in a gyro-averaged sense for obliquely propagating waves. To find the condition under which phase trapping could occur, we apply $\mathrm{d} / \mathrm{d} t$ to Eq. (15) giving

$\ddot{\zeta}=-\dot{\Omega}-\dot{\omega}+\ddot{\lambda}+\frac{\mathrm{d}}{\mathrm{d} t}\left(k_{\|} v_{\|}\right)$,

where $\lambda \equiv \int k_{\mathrm{x}} d x=\left[k_{\mathrm{x}} v_{\perp} /(-\Omega)\right] \sin \theta=k_{\mathrm{x}} \rho_{\perp} \sin \theta=\beta \sin \theta$ to the lowest order in $\epsilon$. We have assumed that $k_{\mathrm{x}} \rho_{\perp}$ does not vary significantly during one gyroperiod. Expanding the last term on the right hand side of the above equation, we have

$\ddot{\zeta}=-\dot{\Omega}-\dot{\omega}+\ddot{\lambda}+\dot{k}_{\|} v_{\|}+k_{\|} \dot{v}_{\|}$.

For interactions near the $l_{0}$-order resonance so that $l_{0} \Omega+$ $k_{\|} v_{\|}-\omega \simeq 0$ is satisfied, we define $\eta=\zeta-\lambda-\left(l_{0}+1\right) \theta$ so that $\dot{\eta}=l_{0} \Omega+k_{\|} v_{\|}-\omega$ and

$\ddot{\eta}=l_{0} \dot{\Omega}+\dot{k}_{\|} v_{\|}+k_{\|} \dot{v}_{\|}-\dot{\omega}$.

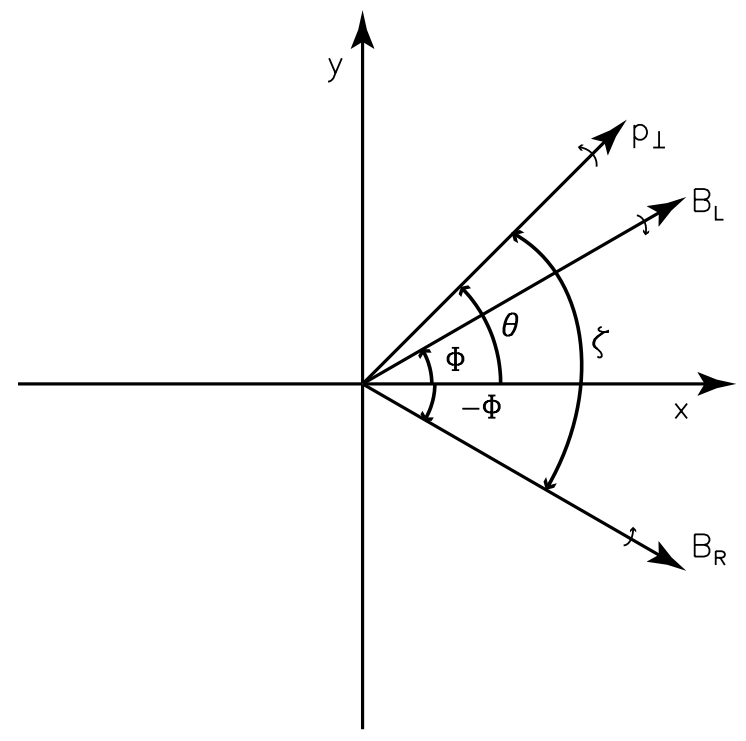

Fig. 1. An illustration of the relative positions of wave fields $\boldsymbol{B}_{\mathrm{R}}$ and $\boldsymbol{B}_{\mathrm{L}}$ and particle's perpendicular momentum $\boldsymbol{p}_{\perp}$.

Here the time derivative of $\Omega$ and $v_{\|}$could be found simply from their definitions as

$$
\begin{aligned}
& \dot{\Omega} \simeq v_{\|} \frac{\partial \Omega}{\partial z}-\Omega \frac{\dot{\gamma}}{\gamma}, \\
& \dot{v}_{\|}=\frac{\dot{p}_{\|}}{\gamma m}-v_{\|} \frac{\dot{\gamma}}{\gamma} .
\end{aligned}
$$

The time rate of change of $\gamma$ in Eqs. (26) and (27) could be found from the dot product of $\boldsymbol{p}$ and Eq. (13), which gives

$\frac{\mathrm{d} p^{2}}{\mathrm{~d} t}=2 q \boldsymbol{E}_{\mathrm{w}} \cdot \boldsymbol{p}$,

and this leads to

$\frac{\mathrm{d} \gamma^{2}}{\mathrm{~d} t}=\frac{\mathrm{d} p^{2}}{\mathrm{~d} t} \frac{1}{m^{2} c^{2}}=2 q \frac{\boldsymbol{E}_{\mathrm{w}} \cdot \boldsymbol{p}}{m^{2} c^{2}}$

by the use of $\gamma^{2}=1+p^{2} / m^{2} c^{2}$. Consequently,

$$
\begin{aligned}
\frac{\mathrm{d} \gamma}{\mathrm{d} t}= & \frac{q}{\gamma} \frac{\boldsymbol{E}_{\mathrm{w}} \cdot \boldsymbol{p}}{m^{2} c^{2}}=\frac{q}{\gamma m^{2} c^{2}} \\
& {\left[-p_{\|} E_{\mathrm{Z}}^{\mathrm{W}} \sin \Phi-E_{\mathrm{R}}^{\mathrm{W}} p_{\perp} \sin \zeta+E_{\mathrm{L}}^{\mathrm{W}} p_{\perp} \sin (\zeta-2 \Phi)\right] . }
\end{aligned}
$$

Substituting Eqs. (26) and (27) into Eq. (25) and using (30) gives us

$\ddot{\eta}=h(\boldsymbol{r}, t)+C_{1} \sin \Phi+C_{2} \sin \zeta+C_{3} \sin (\zeta-2 \Phi)$,

where

$C_{1}=\left(l_{0} \Omega+k_{\|} v_{\|}\right) \frac{q}{\gamma^{2} m^{2} c^{2}} p_{\|} E_{\mathrm{z}}^{\mathrm{w}}-\frac{k_{\|}}{\gamma m} q E_{\mathrm{z}}^{\mathrm{w}}$, 
$C_{2}=\left(l_{0} \Omega+k_{\|} v_{\|}\right) \frac{q}{\gamma^{2} m^{2} c^{2}} p_{\perp} E_{\mathrm{R}}^{\mathrm{w}}-\frac{k_{\|}}{\gamma m} p_{\perp} \omega_{\mathrm{R}}$,

$C_{3}=-\left(l_{0} \Omega+k_{\|} v_{\|}\right) \frac{q}{\gamma^{2} m^{2} c^{2}} p_{\perp} E_{\mathrm{L}}^{\mathrm{w}}-\frac{k_{\|}}{\gamma m} p_{\perp} \omega_{\mathrm{L}}$,

and

$h(\boldsymbol{r}, t)=l_{0} v_{\|} \frac{\partial \Omega}{\partial z}+\dot{k}_{\|} v_{\|}-\dot{\omega}+A_{1} \frac{k_{\|}}{\gamma m}$.

Making use of the Bessel function identity

$\mathrm{e}^{\mathrm{i} \beta \sin \theta}=\sum_{l=-\infty}^{\infty} J_{l}(\beta) \mathrm{e}^{\mathrm{i} l \theta}$

where $J_{l}(\beta)$ is the $l$-th order Bessel function of the first kind with argument $\beta$, we have

$$
\begin{aligned}
\ddot{\eta}= & h(\boldsymbol{r}, t)+C_{1} \sum_{l} J_{l}(\beta) \sin \left[\eta+\left(l+l_{0}\right) \theta\right] \\
& +C_{2} \sum_{l} J_{l}(\beta) \sin \left[\eta+\left(l+l_{0}+1\right) \theta\right] \\
& -C_{3} \sum_{l} J_{l}(\beta) \sin \left[\eta+\left(l+l_{0}-1\right) \theta\right] .
\end{aligned}
$$

Averaging over the rapidly oscillating gyrophase angle $\theta$ and using that $\eta \simeq\langle\eta\rangle$ through $\mathcal{O}(\epsilon)$ near the $l_{0}$-th order resonance, we have

$\ddot{\eta}+\omega_{t}^{2} \sin \eta=h(z, t)$,

where

$\omega_{t}^{2}=-\left[\left\langle C_{1}\right\rangle J_{-l_{0}}+\left\langle C_{2}\right\rangle J_{-l_{0}-1}-\left\langle C_{3}\right\rangle J_{-l_{0}+1}\right]$

with the argument of Bessel functions $\beta$ and

$h(z, t) \equiv\langle h(\boldsymbol{r}, t)\rangle=l_{0} v_{\|} \frac{\partial \Omega}{\partial z}+\dot{k_{\|}} v_{\|}-\dot{\omega}-\frac{k_{\|}}{2 \Omega} \frac{p_{\perp}^{2}}{(\gamma m)^{2}} \frac{\partial \Omega}{\partial z}$.

Here $\left\langle C_{1}\right\rangle,\left\langle C_{2}\right\rangle$, and $\left\langle C_{3}\right\rangle$ have the same expressions as Eqs. (32)-(34), but with all variables understood to be gyroaveraged.

Equation (38) has the form of a driven pendulum equation. We define a dimensionless parameter $\chi=\left|\omega_{t}^{2}\right| /|h(z, t)|$ to represent the ratio of the wave-induced motion $\left(\omega_{t}^{2}\right)$ and the adiabatic motion $(h(z, t))$. When $\chi<1$, no phase trapping is expected. On the other hand, if $\chi>1$, an electron might be phase trapped so that $\eta$ oscillates around some equilibrium value and the electron stays resonant with the wave, causing large changes of its energy and pitch angle. Previous theory also suggests that chorus waves are generated because of phase trapping of resonant electrons (Omura et al., 2008).

\section{Applications to interactions between electrons and whistler mode waves}

To estimate the importance of nonlinear interactions and to illustrate the use of the dimensionless parameter $\chi$, we consider nonlinear interaction regions (in latitude $\lambda$ and wave normal angle $\psi$ ) for an electron resonant with a constant frequency whistler mode wave at $L=5$ with frequency $\omega=0.1 \Omega_{\mathrm{ce} 0}$, where $\Omega_{\mathrm{ce} 0}$ is the electron gyrofrequency on the equatorial plane. A constant frequency whistler wave could come from VLF ground transmitters. For a discrete chorus element with rising or falling tones (Santolík et al., 2004), the time rate change of $\omega$ should be considered in the calculation (e.g., Omura et al., 2008). For simplicity, we also assume a constant density $\left(n_{\mathrm{e}}=10.0 \mathrm{~cm}^{-3}\right)$ along the magnetic field line. Note that the second term in Eq. (40) is proportional to $\dot{k}_{\|}$. This term is of the order of $\partial \Omega / \partial z$ for a parallel propagating wave with constant frequency, assuming constant electron density along the background magnetic field. However, so far there is no analytical model for calculating general $\dot{k}_{\|}$for oblique waves along a field line. A full treatment of the problem could be conducted in combination of ray-tracing programs to calculate variation of $k_{\|}$along a magnetic field line. In this work, we ignore the $\dot{k}_{\|}$term for illustration purposes. Nonetheless, the main feature of the nonlinear interaction regions are retained, as shown below.

Figure 2 shows the parameter $\chi$ for the main counterstreaming gyroresonance $l_{0}=-1$, for different local pitch angles and wave amplitudes. The $\chi$ is estimated by calculating the absolute value of the ratio of $\omega_{t}^{2}$ (Eq. 39) to $h(z, t)$ (Eq. 40), where the factor $\partial \Omega / \partial z$ is estimated using Earth's dipole magnetic field. We choose the electron's parallel and perpendicular velocities so that the first order resonance condition $\dot{\eta}=-\Omega+k_{\|} v_{\|}-\omega=0$ is always satisfied for each pitch angle, latitude, and wave normal angle. Regions where phase trapping could happen $(\chi>1)$ are indicated by red color. From Fig. 2, several features of phase trapping regions can be seen. First, it is easier for nonlinear interactions to occur near the equatorial region, where the inhomogeneity factor $h(z, t)$ is small. Second, as the wave amplitude increases, the latitude range where phase trapping could happen becomes larger for a given $\alpha$ and $\psi$. At $E=1 \mathrm{MeV}$, the nonlinear effect is important and should be considered for $\alpha=50^{\circ}$ and $70^{\circ}$ when $B_{\mathrm{y}}^{\mathrm{w}}=1 \mathrm{nT}$. Third, the latitude range for phase trapping is larger at $\alpha=50^{\circ}$ than at $10^{\circ}$ and $70^{\circ}$ for a given $\alpha$ and $\psi$, thus it might be easier for nonlinear interactions to occur for electrons with medium pitch angles. This result is consistent with Inan et al. (1978) that deals with nonlinear interactions between nonrelativistic electrons and a parallel propagating wave. Inan et al. (1978) showed that, for the case they considered, $\chi$ is roughly proportional to $\tan \alpha$ when $\alpha$ is small and $(\tan \alpha)^{-1}$ when $\alpha$ is large, with a local maximum at $\alpha \sim 75^{\circ}$ for the parameters they used, if all other parameters are kept constant (see Eq. 9 of (Inan et al., 1978)). Fourth, the particular shape of nonlinear resonance regions when $\alpha=70^{\circ}$ contains periodic "nulls" in $\psi$, and is related to a phenomenon called "anomalous phase trapping" by Bell (1984). This anomalous phase trapping is caused by the change of sign of $\omega_{t}^{2}$ as explained in Bell (1984). These features are consistent with previous results. 

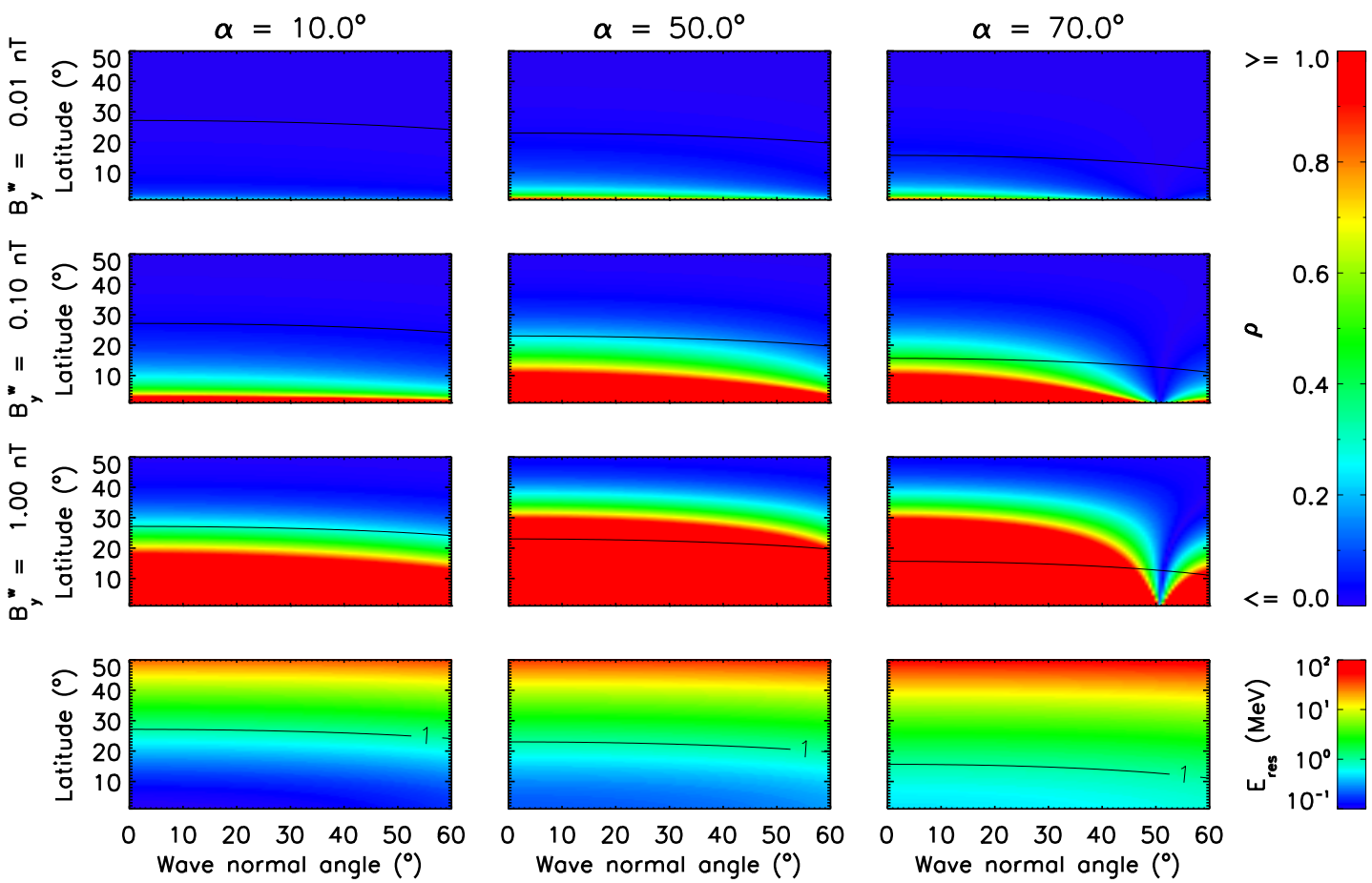

Fig. 2. The nonlinear parameter $\chi$ plotted as a function of latitude and wave normal angle for a whistler wave with $\omega=0.1 \Omega_{\mathrm{ce} 0}$ at selected pitch angles, with effects of time rate change of $k_{\|}$ignored. Here $\Omega_{\mathrm{ce} 0}$ is the electron angular gyrofrequency at the equatorial plane. The first three rows correspond to different wave amplitudes: $B_{\mathrm{y}}^{\mathrm{W}}=0.01 \mathrm{nT}$ (first row), $0.1 \mathrm{nT}$ (second row), and $1.0 \mathrm{nT}$ (third row). Different columns correspond to different local pitch angles: $\alpha=10.0^{\circ}$ (left), $50.0^{\circ}$ (middle), and $70.0^{\circ}$ (right). All black lines show the location of $E_{\text {res }}=1 \mathrm{MeV}$. The fourth row shows the electron resonant energy in $\mathrm{MeV}$.

\section{Discussion and conclusions}

In this work, we derived the equations that describe nonlinear interactions between relativistic electrons and obliquely propagating whistler mode waves from the Lorentz equation of motion. Previous work has been done on nonlinear interactions between nonrelativistic electrons and oblique waves or relativistic electrons and parallel propagating waves, but not both. Thus this work generalizes previous results of Bell (1984) and Omura et al. (2008) and is applicable to interactions between relativistic radiation belt electrons and oblique whistler mode waves.

Using a simplified model and ignoring effects of $\dot{k}_{\|}$, we showed potential regions of nonlinear interactions between electrons and a constant frequency whistler wave. The main features we found are consistent with previous results, and nonlinear interactions might be important for electrons even with moderate amplitude whistler waves $(0.1 \mathrm{nT})$ near the equatorial region. In general, the nonlinear parameter $\chi$ depends on wave amplitude, background inhomogeneity, particle's pitch angle, and the time variations of $\omega$ and $k_{\|}$.

Effects of $\dot{k}_{\|}$should be considered in a more careful evaluation of nonlinear interaction regions with the aid of a raytracing program. The overall importance of nonlinear interactions in radiation belt dynamics is currently under intensive research from the point of view of both theory and observation. Furthermore, phase trapping is generally considered to be important in the generation of chorus waves. Thus understanding the nonlinear interactions is an important step to better understand the dynamics of radiation belts.

Acknowledgements. This research was supported at UCLA by NSF grant 0903802, which was awarded through the NSF/DOE Plasma Partnership program.

Edited by: B. Tsurutani

Reviewed by: G. S. Lakhina and O. Verkhoglyadova

\section{References}

Albert, J. M.: Cyclotron resonance in an inhomogeneous magnetic field, Phys. Fluids B, 5, 2744-2750, doi:10.1063/1.860715, 1993.

Albert, J. M.: Diffusion by one wave and by many waves, J. Geophys. Res., 115, A00F05, doi:10.1029/2009JA014732, 2010.

Albert, J. M., Meredith, N. P., and Horne, R. B.: Three-dimensional diffusion simulation of outer radiation belt electrons during the 9 October 1990 magnetic storm, J. Geophys. Res., 114, A09214, doi:10.1029/2009JA014336, 2009. 
Baker, D. N., Blake, J. B., Callis, L. B., Cummings, J. R., Hovestadt, D., Kanekal, S., Klecker, B., Mewaldt, R. A., and Zwickl, R. D.: Relativistic electron acceleration and decay time scales in the inner and outer radiation belts: SAMPEX, Geophys. Res. Lett., 21(6), 409-412, doi:10.1029/93GL03532, 1994.

Baker, D. N., Li, X., Turner, N., Allen, J. H., Bargatze, L. F., Blake, J. B., Sheldon, R. B., Spence, H. E., Belian, R. D., Reeves, G. D., Kanekal, S. G., Klecker, B., Lepping, R. P., Ogilvie, K., Mewaldt, R. A., Onsager, T., Singer, H. J., and Rostoker, G.: Recurrent geomagnetic storms and relativistic electron enhancements in the outer magnetosphere: ISTP coordinated measurements, J. Geophys. Res., 102, 14141-14148, 1997.

Bell, T. F.: The nonlinear gyroresonance interaction between energetic electrons and coherent VLF waves propagating at an arbitrary angle with respect to the Earth's magnetic field, J. Geophys. Res., 89, 905-918, 1984.

Bortnik, J. and Thorne, R.: The dual role of ELF/VLF chorus waves in the acceleration and precipitation of radiation belt electrons, J. Atmos. Sol.-Terr. Phy., 69, 378-386, doi:10.1016/j.jastp.2006. 05.030, 2007.

Bortnik, J., Thorne, R. M., and Inan, U. S.: Nonlinear interaction of energetic electrons with large amplitude chorus, Geophys. Res. Lett., 35, L21102, doi:10.1029/2008GL035500, 2008.

Breneman, A. W., Kletzing, C. A., Pickett, J., Chum, J., and Santoík, O.: Statistics of multispacecraft observations of chorus dispersion and source location, J. Geophys. Res., 114, A06202, doi:10.1029/2008JA013549, 2009.

Cattell, C., Wygant, J. R., Goetz, K., Kersten, K., Kellogg, P. J., von Rosenvinge, T., Bale, S. D., Roth, I., Temerin, M., Hudson, M. K., Mewaldt, R. A., Wiedenbeck, M., Maksimovic, M., Ergun, R., Acuna, M., and Russell, C. T.: Discovery of very large amplitude whistler-mode waves in Earth's radiation belts, Geophys. Res. Lett., 35, L01105, doi:10.1029/2007GL032009, 2008.

Chum, J., Santoík, O., Gurnett, D. A., and Pickett, J. S.: Oblique lower band chorus waves: Time shifts between discrete elements observed by the Cluster spacecraft, J. Geophys. Res., 114, A00F02, doi:10.1029/2009JA014366, 2009.

Cully, C. M., Bonnell, J. W., and Ergun, R. E.: THEMIS observations of long-lived regions of large-amplitude whistler waves in the inner magnetosphere, Geophys. Res. Lett., 35, L17S16, doi: 10.1029/2008GL033643, 2008.

Dysthe, K. B.: Some studies of triggered whistler emissions, J. Geophys. Res., 76, 6915-6931, 1971.

Friedel, R. H. W., Reeves, G. D., and Obara, T.: Relativistic electron dynamics in the inner magnetosphere: a review, J. Atmos. Sol.Terr. Phy., 64, 265-282, 2002.

Furuya, N., Omura, Y., and Summers, D.: Relativistic turning acceleration of radiation belt electrons by whistler mode chorus, J. Geophys. Res., 113, A04224, doi:10.1029/2007JA012478, 2008.

Helliwell, R. A.: A theory of discrete VLF emissions from the magnetosphere, J. Geophys. Res., 72, 4773-4790, 1967.

Horne, R. B. and Thorne, R. M.: Potential waves for relativistic electron scattering and stochastic acceleration during magnetic storms, Geophys. Res. Lett., 25, 3011-3014, 1998.

Horne, R. B., Thorne, R. M., Glauert, S. A., Albert, J. M., Meredith, N. P., and Anderson, R. R.: Timescale for radiation belt electron acceleration by whistler mode chorus waves, J. Geophys. Res., 110, A03225, doi:10.1029/2004JA010811, 2005a.
Horne, R. B., Thorne, R. M., Shprits, Y. Y., Meredith, N. P., Glauert, S. A., Smith, A. J., Kanekal, S. G., Baker, D. N., Engebretson, M. J., Posch, J. L., Spasojevic, M., Inan, U. S., Pickett, J. S., and Decreau, P. M. E.: Wave acceleration of electrons in the Van Allen radiation belts, Nature, 437, 227-230, doi: 10.1038/nature03939, 2005b.

Inan, U. S., Bell, T. F., and Helliwell, R. A.: Nonlinear pitch angle scattering of energetic electrons by coherent VLF waves in the magnetosphere, J. Geophys. Res., 83, 3235-3253, 1978.

Inan, U. S., Platino, M., Bell, T. F., Gurnett, D. A., and Pickett, J. S.: Cluster measurements of rapidly moving sources of ELF/VLF chorus, J. Geophys. Res., 109, A05214, doi:10.1029/ 2003JA010289, 2004.

Kennel, C. F. and Engelmann, F.: Velocity space diffusion from weak plasma turbulence in a magnetic field, Phys. Fluids, 9, 2377, doi:10.1063/1.1761629, 1966.

Nunn, D., Omura, Y., Matsumoto, H., Nagano, I., and Yagitani, S.: The numerical simulation of VLF chorus and discrete emissions observed on the Geotail satellite using a Vlasov code, J. Geophys. Res., 102, 27083-27097, 1997.

Omura, Y., Nunn, D., Matsumoto, H., and Rycroft, M.: A review of observational, theoretical and numerical studies of VLF triggered emissions, J. Atmos. Sol.-Terr. Phy., 53, 351-368, doi:10.1016/ 0021-9169(91)90031-2, 1991.

Omura, Y., Katoh, Y., and Summers, D.: Theory and simulation of the generation of whistler-mode chorus, J. Geophys. Res., 113, A04223, doi:10.1029/2007JA012622, 2008.

Omura, Y., Hikishima, M., Katoh, Y., Summers, D., and Yagitani, S.: Nonlinear mechanisms of lower-band and upper-band VLF chorus emissions in the magnetosphere, J. Geophys. Res., 114, A07217, doi:10.1029/2009JA014206, 2009.

Reeves, G. D., McAdams, K. L., Friedel, R. H. W., and O'Brien, T. P.: Acceleration and loss of relativistic electrons during geomagnetic storms, Geophys. Res. Lett., 30(10), 1529, doi:10.1029/2002GL016513, 2003.

Santolík, O., Gurnett, D. A., Pickett, J. S., Parrot, M., and Cornilleau-Wehrlin, N.: Spatio-temporal structure of stormtime chorus, J. Geophys. Res., 108, 1278, doi:10.1029/ 2002JA009791, 2003.

Santolík, O., Gurnett, D. A., Pickett, J. S., Parrot, M., and Cornilleau-Wehrlin, N.: A microscopic and nanoscopic view of storm-time chorus on 31 March 2001, Geophys. Res. Lett., 31, L02801, doi:10.1029/2003GL018757, 2004.

Stix, T. H.: The Theory of Plasma Waves, McGraw-Hill, 1962.

Trakhtengerts, V. Y.: Magnetosphere cyclotron maser: Backward wave oscillator generation regime, J. Geophys. Res., 100, 17205-17210, 1995.

Tsurutani, B. T., Verkhoglyadova, O. P., Lakhina, G. S., and Yagitani, S.: Properties of dayside outer zone chorus during HILDCAA events: Loss of energetic electrons, J. Geophys. Res., 114, A03207, doi:10.1029/2008JA013353, 2009.

Varotsou, A., Boscher, D., Bourdarie, S., Horne, R. B., Meredith, N. P., Glauert, S. A., and Friedel, R. H.: Three-dimensional test simulations of the outer radiation belt electron dynamics including electron-chorus resonant interactions, J. Geophys. Res., 113, A12212, doi:10.1029/2007JA012862, 2008. 